Article

\title{
Bone Chemical Composition Assessment with Multi-Wavelength Photoacoustic Analysis
}

\author{
Ting Feng 1,2,3,4®D, Yunhao Zhu ${ }^{3,4}$, Kenneth M. Kozloff ${ }^{4}$, Basma Khoury ${ }^{4}$, Yejing Xie ${ }^{1}$, \\ Xueding Wang ${ }^{4}$, Meng Cao ${ }^{3}$, Jie Yuan ${ }^{3, * \mathbb{D}}$, Dean Ta ${ }^{5}$ and Qian Cheng ${ }^{2, * \mathbb{C}}$ \\ 1 School of Electronics and Optical Engineering, Nanjing University of Science and Technology, \\ Nanjing 210094, China; fengting@njust.edu.cn (T.F.); xieyejing@njust.edu.cn (Y.X.) \\ 2 Institute of Acoustics, School of Physics Science and Engineering, Tongji University, Shanghai 200092, China \\ 3 Department of Electronic Science and Engineering, Nanjing University, Nanjing 210023, China; \\ yunhaoz@umich.edu (Y.Z.); caomeng@smail.nju.edu.cn (M.C.) \\ 4 Department of Biomedical Engineering, University of Michigan, Ann Arbor, MI 48019, USA; \\ kenkoz@umich.edu (K.M.K.); khouryb@med.umich.edu (B.K.); xdwang@umich.edu (X.W.) \\ 5 Department of Electronic Engineering, Fudan University, Shanghai 200433, China; tda@fudan.edu.cn \\ * Correspondence: yuanjie@nju.edu.cn (J.Y.); q.cheng@tongji.edu.cn (Q.C.)
}

Received: 4 September 2020; Accepted: 12 November 2020; Published: 19 November 2020

\begin{abstract}
In this study, the feasibility of assessing the chemical composition in bone using the multi-wavelength photoacoustic analysis (MWPA) method was investigated. By illuminating a bone specimen using laser light with a wavelength tunable over an optical spectrum from $680 \mathrm{~nm}$ to $950 \mathrm{~nm}$, the optical absorption spectrum of the bone was acquired. Then, with the optical absorption spectra of all the optically absorbing chemical components in the bone known, a spectral unmixing procedure was performed to quantitatively assess the relative content of each chemical component. The experimental results from porcine rib bones demonstrated that the contents of the chemical components, including not only non-organic materials such as minerals and water but also organic materials including oxygenated hemoglobin, deoxygenated hemoglobin, lipid, and collagen, can all be assessed by MWPA. As the chemical composition in the bone is directly associated with functional and metabolic activities, the finding from this study suggests that the MWPA method could offer a new diagnostic tool for the non-invasive evaluation of bone health.
\end{abstract}

Keywords: photoacoustic; bone; multi-wavelength photoacoustic analysis; osteoporosis; chemical composition

\section{Introduction}

The number of patients with osteoporosis, a serious public health threat with significant physical, psychological, and economic impacts, is expected to grow, mainly due to the increase in the aging population worldwide. In osteoporosis, the bone mineral density (BMD) is reduced, bone microarchitecture (BMA) deteriorates, and the amount and variety of proteins in bone are altered. Currently, most clinically used non-invasive assessment methods are based on the use of X-ray or ultrasound [1,2]. These methods, although often used to measure bone mineral density (BMD) and some mechanical properties, have limited sensitivity to monitor the chemical or molecular changes in the bone that result from disease or aging. In addition, X-ray-based techniques use ionizing radiation, which is not ideal for pediatric applications or long-term repetitive monitoring. Quantitative ultrasound (QUS) technologies provide practical and low-cost alternatives, and have already led to clinical instrumentations [3,4]. The QUS bone assessment methods are usually based on the measurements of sound velocity (SOS) and broadband ultrasound attenuation (BUA) through a 
given tissue. However, the specificity of QUS is limited when pathogenic bone diseases are determined by microstructure and chemical changes rather than the change in bone mass [5-7].

Bone quantity and quality are dependent on not only the mass and structure of the non-organic mineral matrix but also the organic matrix which is associated with the bone blood flow and cellular metabolism. The main components of the organic matrix in the bone are lipid, blood cells, collagen, and proteins. It is the mineral component of the non-organic matrix that makes bone hard and rigid, but the arrangement of the collagen fibers in the organic matrix makes bone strong. In addition, the other organic components, including bone marrow fat, marrow perfusion, and oxygen saturation, are related to the cellular metabolism of bone. Therefore, those features of chemical components may offer newly detectable information for the early diagnosis of osteoporosis [8,9]. Recently, it has been reported that magnetic resonance imaging (MRI) can distinguish changes in bone marrow lipid content and bone microarchitecture between normal bone and osteopenic bone [10-12]. Due to its high cost and complexity, MRI examinations are not a feasible replacement for routine X-ray-based methods. In prior studies, optical spectroscopic techniques have been used to evaluate how alterations of bone composition contribute to bone quality changes related to aging, disease, or injury [13-16]. Traditional optical techniques, however, suffer from low spatial resolution and limited detection specificity due to the overwhelming optical scattering in biological tissues.

The emerging biomedical photoacoustic (PA) techniques have the unique capability to probe the highly sensitive optical absorption contrast in deep biological tissues [17,18]. The PA signal generated by the bone contains both microstructural information and molecular and chemical information, both highly relevant to bone health. In previous research, Lashkari et al. evaluated the cortical and trabecular bone structure and density variations in bone using a dual backscattered ultrasound and PA radar system $[19,20]$. Our group has studied the feasibility of using the thermal photoacoustic (TPA) method and photoacoustic spectral analysis (PASA) method to assess the BMD and BMA of trabecular bones in rat models [21-23]. Cayla et al. introduces an optimized PA imaging technique to assess $\mathrm{SO}_{2}$ within the femoral bone marrow cavity through disease progression in a murine model [24]. Recently, Idan Steinberg et al. used the dual-modality multispectral PA system to quantify the blood over fat ratio present in the marrow, which was correlated with the molecular changes in the long bone [25].

This study aimed at validating the feasibility of the multi-wavelength PA analysis (MWPA) method in quantifying the chemical information in the trabecular bones with differing BMD. The experiments were conducted on fresh and ethylenediaminetetraacetic acid (EDTA)-treated porcine ribs. The PA spectroscopic curves of the rib bones with different BMDs were measured. Then, with the optical absorption spectra of all the relevant chemical components known, including hydroxyapatite, lipid, deoxygenated hemoglobin, oxygenated hemoglobin, collagen, and water, a spectral unmixing procedure was conducted, which quantified the relative content of each chemical component in the bone. To validate this method, the measurements from the bone specimens with different BMDs were compared.

\section{Materials and Methods}

\subsection{Experimental Setup}

Each bone sample was measured by using MWPA over an optical spectrum from $680 \mathrm{~nm}$ to $950 \mathrm{~nm}$ with a constant interval of $10 \mathrm{~nm}$ (in total, 28 wavelengths). The experimental setup used to study the chemical components of bone is shown in Figure 1. The light beam from a Nd:YAG laser pumped optical parametric oscillator (OPO) (Vibrant B, Opotek, Carlsbad, CA, USA) illuminated the bone with a beam diameter of $4 \mathrm{~mm}$. The light fluence at each wavelength in the entire optical spectrum was controlled to be $15-20 \mathrm{~mJ} / \mathrm{cm}^{2}$ per pulse, which was lower than the safety limit of the American National Standards Institute (ANSI). After passing through a beam splitter, $10 \%$ of the laser energy was guided to a black rubber. The PA signal generated from the black rubber 
was recorded by an ultrasound transducer (V310-SU, Olympus, Center Valley, PA, USA) which was later used as a reference for calibrating the PA signal from the bone. Ninety percent of the laser energy was guided to the bone sample which was immersed in a water bath for acoustic coupling. The generated PA signal from the bone was received by an ultrasonic transducer ( $\mathrm{FC}=5 \mathrm{MHz}, \mathrm{V} 326-\mathrm{SU}$, Olympus, Center Valley, PA, USA). The detected PA signal was amplified, then digitized by a digital oscilloscope (TDS 540, Tektronix, Inc., Beaverton, OR, USA), and then collected by a PC. To enhance the signal-to-noise ratio (SNR), the PA signal was averaged over 50 laser pulses.

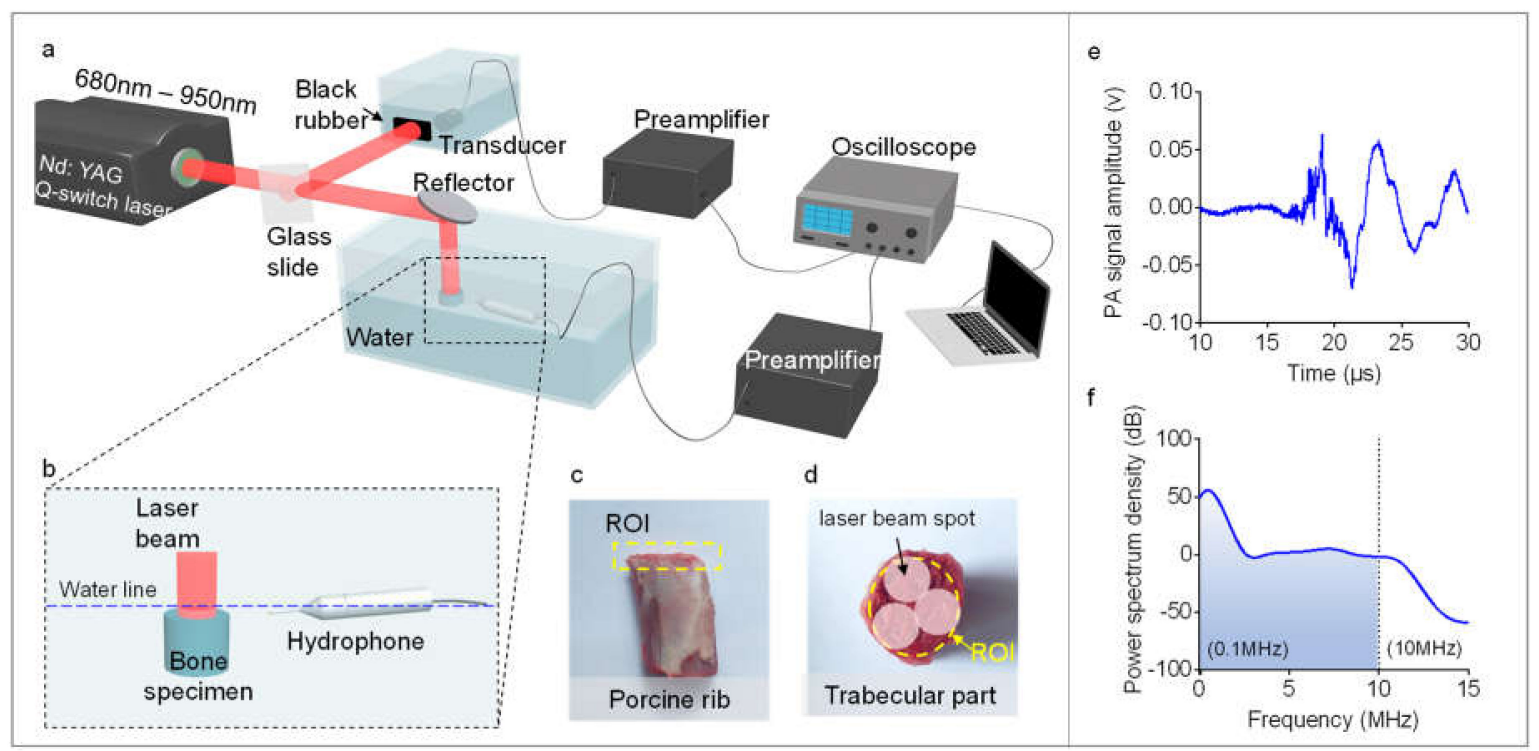

Figure 1. Experimental setup. (a,b) The schematic diagram of the experiment setup. (c,d) The photographs of the bone samples. ROI stands for the regions of interest. (e) An example of the photoacoustic (PA) signal generated by the bone specimen. (f) An example of the power spectrum density (PSD) of the PA signal generated by the bone.

\subsection{Bone Specimens}

To assess MWPA measurements in a clinically relevant animal model, fresh porcine rib bones were obtained from a local grocery store, as shown in Figure 1c,d. Three groups of bones were studied, each with five specimens $(n=5)$, including Group 1 for untreated bones, Group 2 for bones treated with EDTA for $13 \mathrm{~h}$, and Group 3 for bones treated with EDTA for $26 \mathrm{~h}$. According to the literature [26-28], bone can be decalcified by EDTA solution $(0.5 \mathrm{M})$, while the organic materials in the bone will stay. Based on our observation, however, the blood in the bone was also partly lost after the bone was immersed in the EDTA solution for a certain period of time. To confirm the decalcification of the bones after being treated with EDTA, each bone was imaged by a Faxitron microradiograph (see Figure 2a-c). The images were further processed using Matlab software to evaluate the mean grayscale value for each sample as an indicator of the BMD in the bone. The mean and standard division of the gray values are $125.20 \pm(9.143), 77.41 \pm(15.99), 74.17 \pm(15.48)$, as shown in Figure 2d, for Groups 1, 2, and 3, respectively. Unpaired $t$-tests were conducted to compare the grayscale values among the three groups, with a null hypothesis that there was no statistically significant difference between any two of the three groups. $p$-values of 0.03 and 0.02 were archived between Group 1 and Group 2, and also between Group 1 and Group 3, respectively, demonstrating that the average BMD for untreated bones in Group 1 was significantly larger than those in the demineralized bones in Group 2 and Group 3. No statistically significant difference was observed between Group 2 and Group 3, although the average BMD of Group 3 was slightly lower than that of Group 2. 

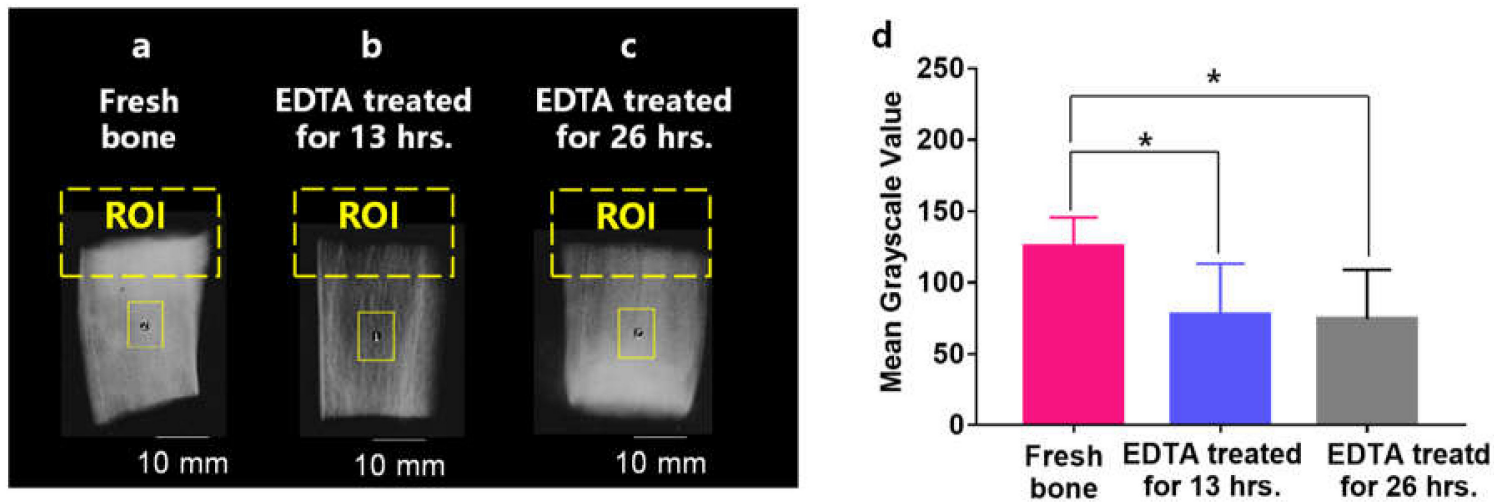

Figure 2. X-ray imaging results for three bone groups. (a) The X-ray image of an untreated bone from Group 1. (b) The X-ray image of a bone from Group 2 treated with EDTA for $13 \mathrm{hrs}$. (c) The X-ray image of a bone in Group 3 treated with EDTA for $26 \mathrm{~h}$. (d) The grayscale value for the three bone groups; * stands for $p<0.05$.

\subsection{Theory}

The amplitude of the PA signal generated by the bone after ignoring the reflected acoustic wave from the boundary can be expressed as $[29,30]$ :

$$
p(r, \lambda)=k \cdot \Gamma \cdot \eta_{t h} \cdot \mu_{a}(\lambda) \cdot F_{0} \cdot e^{-\mu_{e f f}(\lambda) \cdot r} \cdot e^{-\alpha \cdot\left(z_{0}-r\right)}
$$

where $\Gamma$ is the Grüneisen parameter, which can be expressed as $\Gamma=\frac{\beta \cdot c^{2}}{C_{p}}$, where $\beta$ is the thermal coefficient of volume expansion, $c$ is the SOS of tissue, and $C_{p}$ is the heat capacity at constant pressure; $k$ is a constant accounting for the impulse response of the detection system; $\eta_{t h}$ is the heat conversion efficiency; $\mu_{a}$ is optical absorption of bone tissue; $F_{0}$ is the light fluence generated by the laser; $\mu_{e f f}$ is the effective optical attenuation coefficient in the bone; $e^{-\mu_{e f f}(\lambda) \cdot r}$ is the light attenuation in the bone; $\alpha$ is the ultrasound attenuation coefficient in the bone; $e^{-\alpha \cdot\left(z_{0}-r\right)}$ is the ultrasound attenuation in the bone. Since both the ultrasound and optical attenuation are large in bone tissue $[4,31,32]$, the PA signal received by the transducer is mostly generated from the bone surface $(r=0)$. Then, the amplitude of the PA signal received by the transducer could be simplified as

$$
p(\lambda)=k \cdot \Gamma \cdot \eta_{t h} \cdot \mu_{a}(\lambda) \cdot F_{0} \cdot e^{-\alpha \cdot z_{0}} .
$$

In this equation, $F_{0}$ can be calibrated by the recorded laser energy at different wavelengths, while $k$, $\Gamma, \eta_{t h}$, and $\alpha$ are independent of the wavelength. Therefore, $k, \Gamma, \eta_{t h}$, and $\alpha$ can be removed by dividing $\mu_{a}\left(\lambda_{0}\right)$ at the reference wavelength of $\lambda_{0}$ :

$$
\frac{p(\lambda)}{p\left(\lambda_{0}\right)}=\frac{k \cdot \Gamma \cdot \eta_{t h} \cdot \mu_{a}(\lambda) \cdot F_{0} \cdot e^{-\alpha \cdot z_{0}}}{k \cdot \Gamma \cdot \eta_{t h} \cdot \mu_{a}\left(\lambda_{0}\right) \cdot F_{0} \cdot e^{-\alpha \cdot z_{0}}}
$$

From this equation, we can get the $\mu_{a}(\lambda)$ by using

$$
\frac{\mu_{a}(\lambda)}{\mu_{a}\left(\lambda_{0}\right)}=\frac{p(\lambda)}{p\left(\lambda_{0}\right)}
$$

Equation (4) indicates that, by performing multi-wavelength PA measurement of bone samples, we can measure the relative optical absorption spectrum in the trabecular bone. With the relative optical absorption spectrum of a bone measured, spectral unmixing was then be performed to derive the relative contribution of each chemical component to the optical absorption spectrum [33]. A least-squares method was used, which tries to fit the PA measured optical absorption spectrum of bone via a linear 
combination of the optical spectra of all the relevant chemical components leading to the minimum sum of the squared residuals of the measurement points from the fitting line.

\subsection{Signal Processing}

The processing of the multi-wavelength PA signals from each bone specimen follows the steps listed below. Step 1: the amplitude of the PA signal acquired from the bone was divided by the amplitude of the PA signal from the black rubber for calibration. This calibration not only normalized the outputs of laser energy at different wavelengths, but also removed the variation due to the pulse-to-pulse fluctuation of the laser energy. Step 2: the PA signal was transferred to the frequency domain via fast Fourier transform; then the PA frequency power was integrated from $0.5 \mathrm{MHz}$ to $10 \mathrm{MHz}$. The integrated power reflects the strength of the PA bone signal in the frequency range of $0.5-10 \mathrm{MHz}$ without being affected by the low frequency ( $<0.5 \mathrm{MHz})$ noise or high frequency $(>10 \mathrm{MHz})$ noise. Step 3: the Steps 1 and 2 were repeated for the PA signals at all the laser wavelengths over the optical spectrum from $680 \mathrm{~nm}$ to $950 \mathrm{~nm}$. Then a curve representing the integrated PA power as a function of the laser wavelength was generated. This curve, namely the PA spectrum, represents the spectroscopic optical absorption of the bone measured by the multi-wavelength PA method. Step 4: In order to improve the stability and reduce the measurement error, each bone specimen was detected from three different orientations. Then, the Steps 1, 2, and 3 were repeated, and the PA spectrum curves from three different orientations were obtained and averaged for further analyses. Step 5: by performing spectral unmixing using the least-squares method, the relative contents of all the optically absorbing chemical components in the bone were calculated from the PA spectrum acquired from $680 \mathrm{~nm}$ to $950 \mathrm{~nm}$.

\section{Results and Discussion}

Fresh human bone contains non-organic mineral (hydroxyapatite) and water, as well as organic compounds, including collagen, lipid, and hemoglobin [34-36]. Each non-organic and organic material in the bone has its own unique optical absorption spectrum, and may contribute to the PA signal from the bone. In the spectral range of 680-950 $\mathrm{nm}$, the main optically absorbing components in the bone are hydroxyapatite, oxygenated hemoglobin, deoxygenated hemoglobin, and lipids, while the light absorption of other materials is relatively low $[13,31,37-40]$.

Figure 3 shows the MWPA results for the three groups of bones. The three solid lines in Figure 3a show the averaged PA spectra measured from the three groups of bones, while the standard deviation and error bars are shown by the shaded area next to each curve. An obvious difference can be seen in the PA spectra from the three groups. In the PA spectrum from the fresh bones (Group 1), we can see relatively strong absorption around $700 \mathrm{~nm}, 760 \mathrm{~nm}$, and $860-950 \mathrm{~nm}$. The overall shape of the PA spectrum matches well with results in the literature [13,41]. The optical absorption spectra of the main chemical components in the bone are shown in Figure 3b. We can see that the main characteristics of these absorption spectra, especially for hydroxyapatite, hemoglobin, and lipid, are reflected in the measured PA spectrum from the fresh bone in Figure 3a [21]. We expect that both deoxygenated hemoglobin and hydroxyapatite contribute to the strong absorption in the range of 680-700 nm [42,43]. The peak around $760 \mathrm{~nm}$ in the PA spectrum may come from the absorption of both deoxygenated hemoglobin and lipid [44]. The strong absorption around 900-950 nm may be associated with lipid, oxygenated hemoglobin, and water. According to the literature, lipid has a strong absorption peak at $930 \mathrm{~nm}$, while collagen has an absorption peak near $920 \mathrm{~nm}[44,45]$. However, these peaks at approximately $920-930 \mathrm{~nm}$ can only be noticed in the PA spectrum from the bones treated by EDTA for $26 \mathrm{~h}$ (Group 3). For the other two groups (i.e., Group 1 and Group 2), these peaks cannot be seen, probably because the $900-950 \mathrm{~nm}$ spectral range is still dominated by oxygenated hemoglobin which was not fully removed from the bones in these two groups. 


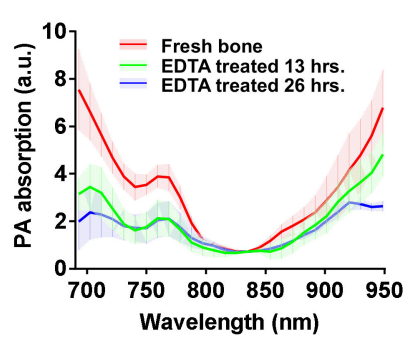

b

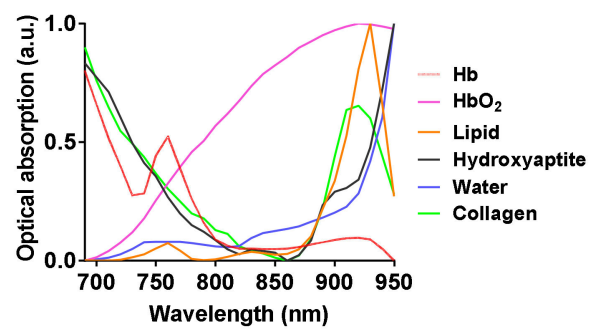

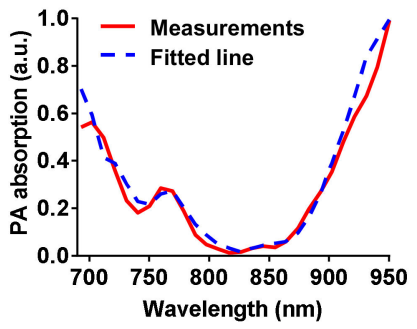

Figure 3. (a) The averaged PA spectra measured from the three groups of porcine ribs, including fresh bones (Group 1), bones treated with EDTA for $13 \mathrm{~h}$ (Group 2), and bones treated with EDTA for $26 \mathrm{~h}$ (Group 3). (b) Optical absorption spectra of major chemical components in the bone, including hydroxyapatite, collagen, oxyhemoglobin $(\mathrm{HbO} 2)$, deoxygenated hemoglobin $(\mathrm{Hb})$, lipids, and water. (c) The averaged PA spectrum from Group 2 (the bones treated with EDTA for $13 \mathrm{~h}$ ) in comparison with the fitted spectrum by using the least-squares method.

With the optical absorption spectra of all the major chemical components in the trabecular bone known [21,31], the relative contribution of each chemical component to the PA spectrum can be derived by performing a spectral unmixing. A least-squares method was used, which tries to fit the PA spectrum via a linear combination of the optical spectra of all the chemical components leading to the minimum sum of the squared residuals of measurement points from the fitting line. As an example, Figure 3c shows the averaged PA spectrum from Group 2 in comparison with the fitted spectrum based on the least-squares method. A very high $\mathrm{R}^{2}$ of 0.97 was achieved, demonstrating that a successful fitting with a high accuracy was achieved in spectral unmixing. For Group 1 and Group 3, the same procedure also led to a satisfactory $\mathrm{R}^{2}$ of 0.96 and 0.94 , respectively.

After the spectral unmixing, the relative contents of the six chemical components in the bone, including hydroxyapatite, deoxygenated hemoglobin, oxygenated hemoglobin, lipid, collagen, and water, were derived, as the results show in Figure 4 for the three groups of bone samples. The spectral unmixing here did not lead to the measurements of molar concentrations of the chemical components because the data in Figure $3 b$ utilized in spectral unmixing were not the molar extinction coefficients. The relative content derived from the spectral unmixing reflects the weight of each optically absorbing chemical component in the measured PA spectral. As expected, when the EDTA treatment time increased, the content of hydroxyapatite decreased [46], while the contents of lipid, collagen, and water increased accordingly. The EDTA treatment also reduced the contents of both oxygenated hemoglobin and deoxygenated hemoglobin, because soaking the bone in the EDTA solution led to the removal of blood from the bone. The bar chart in Figure $4 \mathrm{~b}$ summarizes the changes in mineral (hydroxyapatite) content, blood content (including the two forms of hemoglobin), and the overall content of other materials including lipid, collagen, and water. To examine whether these changes in chemical components in the bone caused by the EDTA treatment are significant, un-paired $t$-tests were performed to compare the results in Figure $4 \mathrm{~b}$. After a 13-hour EDTA treatment and 26-hour EDTA treatment, the mineral content, the blood content, and the overall content of other materials (including lipid, collagen, and water) all show statistically significant changes ( $p$-value $\approx 0.01,0.02,0.01$, respectively). The changes in chemical contents between the two treatment groups (i.e., 13-hour EDTA treatment and 26-hour EDTA treatment) are not significant. 
a

\begin{tabular}{lccc}
\hline & $\begin{array}{c}\text { Fresh bone } \\
\text { (averaged relative } \\
\text { content \%) }\end{array}$ & $\begin{array}{c}\text { Treated 13 hrs. } \\
\text { (averaged relative } \\
\text { content \%) }\end{array}$ & $\begin{array}{c}\text { Treated 26 hrs. } \\
\text { (averaged } \\
\text { relative content \%) }\end{array}$ \\
\hline Mineral (Hydroxyaptite) & 34 & 17 & 12 \\
\hline $\mathrm{Hb}$ & 22 & 12 & 14 \\
\hline $\mathrm{HbO}_{2}$ & 12 & 10 & 6 \\
\hline Lipid & 10 & 14 & 18 \\
\hline Collagen & 9 & 12 & 17 \\
\hline Water & 14 & 14 & 36 \\
\hline Mineral measured by & 100 & 63 & 62 \\
\hline X-ray (Gold standard) & & & \\
\hline
\end{tabular}

b

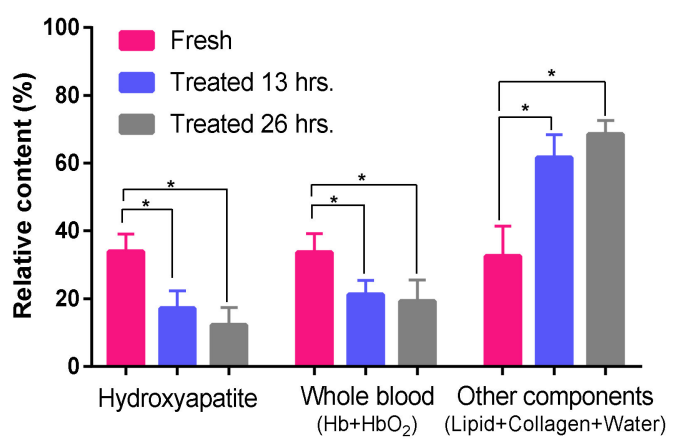

Figure 4. (a) Relative contents of the chemical components in the trabecular bone measured by multi-wavelength photoacoustic analysis (MWPA), including hydroxyapatite, deoxygenated hemoglobin $(\mathrm{Hb})$, oxygenated hemoglobin $\left(\mathrm{HbO}_{2}\right)$, lipid, collagen, and water). (b) The relative contributions of the mineral (hydroxyapatite), whole blood $\left(\mathrm{Hb}+\mathrm{HbO}_{2}\right)$, and other components (lipid + collagen + water) calculated from (a); ${ }^{*}$ stands for $p<0.05$.

Spectral unmixing also enables us to evaluate the contribution from each chemical component to the PA signal from the bone at each specific optical wavelength. The contribution $c_{i}(\lambda)$ for each chemical component was calculated by using

$$
c_{i}(\lambda)=\frac{\left[\mu_{a}(\lambda)\right]_{i} \cdot\left[C_{r}\right]_{i}}{[F(\lambda)]_{i}}
$$

where $\left[\mu_{a}(\lambda)\right]_{i}$ is the optical absorption spectrum of different chemical components at a wavelength of 680-950 nm, $\left[C_{r}\right]_{i}$ is the relative content of different chemical components in the bone, as shown in Figure $3 \mathbf{b}$, and $[F(\lambda)]_{i}$ is the fitting line, as shown in Figure 3c. Based on Equation (5), the quantified contribution $c_{i}(\lambda)$ of different chemical components in the bone can be obtained. Figure 5 shows the quantified contribution $c_{i}(\lambda)$ from each of the six chemical components (i.e., hydroxyapatite, deoxygenated hemoglobin, oxygenated hemoglobin, collagen, lipid, and water) to the bone signal as a function of optical wavelength from $680 \mathrm{~nm}$ to $950 \mathrm{~nm}$. For fresh bones, the major contributors to the PA signals over the studied spectrum were hydroxyapatite, deoxygenated hemoglobin (680-780 nm), and oxygenated hemoglobin $(800-950 \mathrm{~nm}$ ). After EDTA treatment, the contribution from hydroxyapatite was weaker over the entire spectrum; the contribution from oxygenated hemoglobin was almost gone, as not only the blood was removed but it also became older and deoxygenized as a result of the treatment. In addition, because of the loss of both hydroxyapatite and blood after treatment, the relative contributions from lipid, collagen, and water became larger, especially in the spectral range of 900-950 nm. This further explains the peak around 920-930 nm that was observed in the PA spectral curve from the bones treated with EDTA for $26 \mathrm{~h}$, as shown in Figure 3. To indicate the reproducibility, the contribution of each chemical component to the PA signal from the bone as the function of optical wavelength was conducted for each bone sample in those three groups. The average and standard division results of the contribution curves for each group are shown in Figure 5. To examine whether these changes in contribution to the PA signal of different chemical components in the bone caused by the EDTA treatment are significant, a two-way ANOVA test was performed to compare the results in Figure 5a-h. After a 13-hour EDTA treatment and 26-hour EDTA treatment, the contribution of minerals, $\mathrm{HbO}_{2}$, lipid, collagen, and water all show statistically significant changes ( $p$-value $<0.05$ ). The changes in contributions between the two treatment groups (i.e., 13-hour EDTA treatment and 26-hour EDTA treatment) are not significant. 


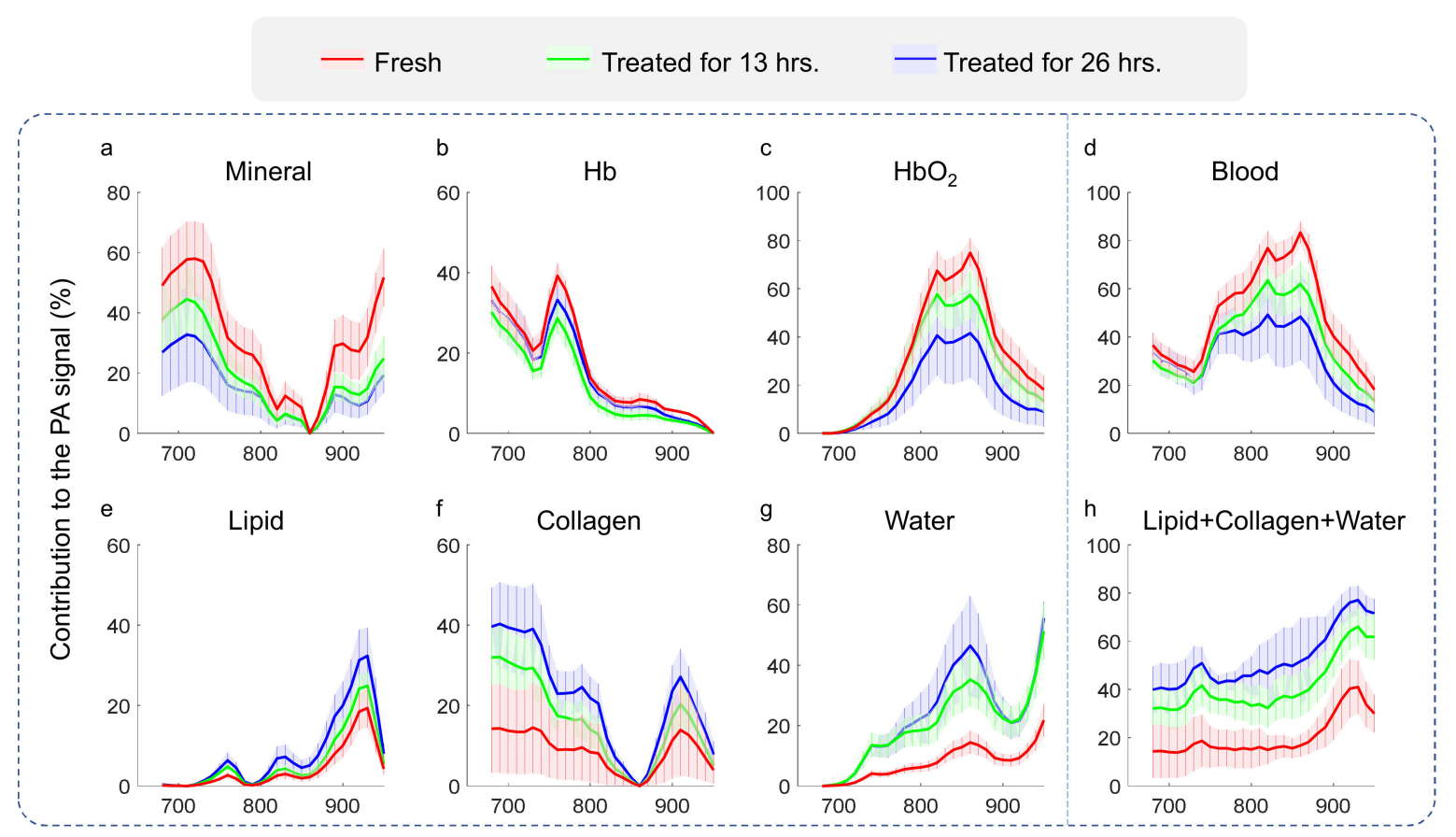

Figure 5. The contributions of each optically absorbing chemical component to the PA absorption curves, including (a) hydroxyapatite, (b) deoxygenated hemoglobin $(\mathrm{Hb}),(\mathbf{c})$ oxygenated hemoglobin $\left(\mathrm{HbO}_{2}\right)$, (d) whole blood $\left(\mathrm{Hb}+\mathrm{HbO}_{2}\right)$, (e) lipid, (f) collagen, (g) water, (h) and the total of lipid, collagen, and water, to the bone PA signals at different optical wavelengths from $680 \mathrm{~nm}$ to $950 \mathrm{~nm}$.

\section{Conclusions}

Via the experiments on clinically relevant porcine rib bones, we studied the feasibility of assessing bone chemical composition by using MWPA method. The quantified relative contents of the chemical components in the three groups of bones, including untreated, treated with EDTA for $13 \mathrm{~h}$, and treated with EDTA for $26 \mathrm{~h}$, were compared, and statistically significant chemical changes due to the treatments were demonstrated. Besides detecting the changes in mineral content in the bone, MWPA could provide additional information of other optically absorbing chemical materials, such as lipid, collagen, oxygenated hemoglobin, deoxygenated hemoglobin, and water. Evaluation of the contents and changes of these organic and non-organic materials in the bone may provide highly valuable information on bone health. In the future, the MWPA could be developed into a low-cost, non-invasive, and patient friendly tool for early diagnosis and longitudinal monitoring of a variety of bone conditions resulting from diseases or aging.

The present study also has some limitations. First, the EDTA bone models do not create changes in bone chemical composition exactly the same as those in osteoporosis or osteopenia bone. Therefore, to pave the way toward clinical applications, extensive studies on clinically relevant animal models of bone diseases followed by clinical trials on human subjects would be necessary to fully understand the performance and limitations of the MWPA method described in this work. Second, the effect of light attenuation was not considered in this study. For in vivo studies, human bones are large, which leads to high light attenuation in them. Therefore, compensation for light attenuation should be considered in future work. Third, for the large ultrasound attenuation in bone for in vivo studies, especially for the high frequency component, therefore, the transducer used for in vivo studies should have a relatively low center frequency. Hence, parts of the future work would be studying the relationship between the PA frequency and the amount of the different chemical components in the bone, as well as the PA spectral curves as functions of the center frequency of the transducer. Fourth, for practical use, the bone assessment technique based on the PA detecting method shown in our manuscript may not available and should be improved with a better design. In particular, 
the mode of detection should be improved based on the target bone in vivo. For example, we used the transmission PA mode for multi-wavelength PA measurement of calcaneus bone in vivo in our recently published study [29]. Fifth, the optical penetration depth at different wavelengths and the ultrasound penetration depth at difference frequencies should be further studied. Based on them, the optimized range of wavelengths and center frequency of the transducer should be used.

Despite these limitations, this study successfully proved the feasibility of using the emerging PA techniques to assess the chemical information in bone. In comparison with established gold standard dual-energy X-ray absorptiometry (DEXA) imaging modalities, the presented PA bone assessment method has many advantages, such as being target specific, non-ionizing, low cost, and patient friendly. Furthermore, the development of PA techniques as a way to accurately quantify organ-level chemical and molecular changes, such as lipid content, blood content (perfusion), and hydroxyapatite content, may allow for early identification of changes in bone metabolism and quality.

Author Contributions: Conceptualization, T.F., J.Y., and Q.C.; methodology, T.F. and Y.Z.; software, T.F., M.C., and Y.X.; validation, K.M.K. and B.K.; formal analysis, T.F.; investigation, T.F.; writing-original draft preparation, T.F.; writing - review and editing, K.M.K., Q.C., J.Y., D.T., and X.W.; visualization, K.M.K.; supervision, J.Y. and Q.C.; project administration, J.Y. and Q.C.; funding acquisition, J.Y., Q.C., and D.T. All authors have read and agreed to the published version of the manuscript.

Funding: This work was supported by the National Key Research and Development Project (No. 2017YFC0111400 \& 2016YFA0100800), National Natural Science Foundation of China (Nos. 11704188 and 11827808, 12034015, $11674249,81702962)$, the Natural Science Foundation of Jiangsu, China (No. BK 20170826), and the Postdoctoral Science Foundation of China under grant No. 2019M651564.

Conflicts of Interest: The authors declare no conflict of interest.

\section{References}

1. Kanis, J.A. Pocket Reference to Osteoporosis; Springer: Cham, Switzerland, 2019; pp. 11-20.

2. Marcocci, C.; Saponaro, F. Multidisciplinary Approach to Osteoporosis; Springer: Cham, Switzerland, 2018; pp. 45-57.

3. Raum, K.; Leguerney, I.; Chandelier, F.; Bossy, E.; Talmant, M.; Saïed, A.; Peyrin, F.; Laugier, P. Bone microstructure and elastic tissue properties are reflected in QUS axial transmission measurements. Ultrasound Med. Biol. 2005, 31, 1225-1235. [CrossRef]

4. Laugier, P. Bone Quantitative Ultrasound; Springer: Cham, Switzerland, 2011; pp. 47-71.

5. Töyräs, J.; Nieminen, M.; Kröger, H.; Jurvelin, J. Bone mineral density, ultrasound velocity, and broadband attenuation predict mechanical properties of trabecular bone differently. Bone 2002, 31, 503-507. [CrossRef]

6. Liu, C.; Ta, D.; Fujita, F.; Hachiken, T.; Matsukawa, M.; Mizuno, K.; Wang, W. The relationship between ultrasonic backscatter and trabecular anisotropic microstructure in cancellous bone. J. Appl. Phys. 2014, 115, 064906. [CrossRef]

7. Njeh, C.; Boivin, C.; Langton, C. The role of ultrasound in the assessment of osteoporosis: A review. Osteoporos. Int. 1997, 7, 7-22. [CrossRef] [PubMed]

8. Justesen, J.; Stenderup, K.; Ebbesen, E.; Mosekilde, L.; Steiniche, T.; Kassem, M. Adipocyte tissue volume in bone marrow is increased with aging and in patients with osteoporosis. Biogerontology 2001, 2, 165-171. [CrossRef] [PubMed]

9. Di Pietro, G.; Capuani, S.; Manenti, G.; Vinicola, V.; Fusco, A.; Baldi, J.; Scimeca, M.; Hagberg, G.E.; Bozzali, M.; Simonetti, G.; et al. Bone marrow lipid profiles from peripheral skeleton as potential biomarkers for osteoporosis: A 1H-MR spectroscopy study. Acad. Radiol. 2016, 23, 273-283. [CrossRef] [PubMed]

10. Griffith, J.F.; Yeung, D.K.W.; Antonio, G.E.; Lee, F.K.H.; Hong, A.W.L.; Wong, S.Y.S.; Lau, E.M.C.; Leung, P.C. Vertebral bone mineral density, marrow perfusion, and fat content in healthy men and men with osteoporosis: Dynamic contrast-enhanced MR imaging and MR spectroscopy. Radiology 2005, 236, 945-951. [CrossRef] [PubMed]

11. Griffith, J.F.; Yeung, D.K.W.; Antonio, G.E.; Wong, S.Y.S.; Kwok, T.C.Y.; Woo, J.; Leung, P.C. Vertebral marrow fat content and diffusion and perfusion indexes in women with varying bone density: MR evaluation. Radiology 2006, 241, 831-838. [CrossRef] 
12. Patsch, J.M.; Burghardt, A.J.; Kazakia, G.; Majumdar, S. Noninvasive imaging of bone microarchitecture. Ann. N. Y. Acad. Sci. 2011, 1240, 77. [CrossRef]

13. Pifferi, A.; Torricelli, A.; Taroni, P.; Bassi, A.; Chikoidze, E.; Giambattistelli, E.; Cubeddu, R. Optical biopsy of bone tissue: A step toward the diagnosis of bone pathologies. J. Biomed. Opt. 2004, 9, 474-481. [CrossRef]

14. Schulmerich, M.V.; Dooley, K.; Morris, M.D.; Vanasse, T.M.; Goldstein, S.A. Transcutaneous fiber optic Raman spectroscopy of bone using annular illumination and a circular array of collection fibers. J. Biomed. Opt. 2006, 11, 060502. [CrossRef] [PubMed]

15. Morris, M.D.; Mandair, G.S. Raman assessment of bone quality. Clin. Orthop. Relat. Res. 2011, 469, $2160-2169$. [CrossRef] [PubMed]

16. Draper, E.R.; Morris, M.D.; Camacho, N.P.; Matousek, P.; Towrie, M.; Parker, A.W.; Goodship, A.E. Novel assessment of bone using time-resolved transcutaneous Raman spectroscopy. J. Bone Miner. Res. 2005, 20, 1968-1972. [CrossRef]

17. Wang, X.; Pang, Y.; Ku, G.; Xie, X.; Stoica, G.; Wang, L.V. Noninvasive laser-induced photoacoustic tomography for structural and functional in vivo imaging of the brain. Nat. Biotechnol. 2003, 21, 803. [CrossRef] [PubMed]

18. Wang, L.V.; Hu, S. Photoacoustic Tomography: In Vivo Imaging from Organelles to Organs. Science 2012, 335, 1458-1462. [CrossRef]

19. Lashkari, B.; Yang, L.; Mandelis, A. The application of backscattered ultrasound and photoacoustic signals for assessment of bone collagen and mineral contents. Quant. Imaging Med. Surg. 2015, 5, 46.

20. Yang, L.; Lashkari, B.; Mandelis, A.; Tan, J.W. Bone Composition Diagnostics: Photoacoustics Versus Ultrasound. Int. J. Thermophys. 2015, 36, 862-867. [CrossRef]

21. Feng, T.; Kozloff, K.M.; Tian, C.; Perosky, J.E.; Hsiao, Y.-S.; Du, S.; Yuan, J.; Deng, C.X.; Wang, X. Bone assessment via thermal photo-acoustic measurements. Opt. Lett. 2015, 40, 1721-1724. [CrossRef]

22. Feng, T.; Perosky, J.E.; Kozloff, K.M.; Xu, G.; Cheng, Q.; Du, S.; Yuan, J.; Deng, C.X.; Wang, X. Characterization of bone microstructure using photoacoustic spectrum analysis. Opt. Express 2015, 23, 25217-25224. [CrossRef]

23. He, W.; Zhu, Y.; Feng, T.; Yuan, J.; Cheng, Q.; Xu, G.; Wang, X. Comparison study of photoacoustic and ultrasound spectrum analysis in osteoporosis detection. Chin. Opt. Lett. 2017, 15, 111101.

24. Wood, C.; Harutyunyan, K.; Sampaio, D.R.; Konopleva, M.; Bouchard, R. Photoacoustic-based oxygen saturation assessment of murine femoral bone marrow in a preclinical model of leukemia. Photoacoustics 2019, 14, 31-36. [CrossRef] [PubMed]

25. Steinberg, I.; Shiloh, L.; Gannot, I.; Eyal, A. First-in-human study of bone pathologies using low-cost and compact dual-wavelength photoacoustic system. IEEE J. Sel. Top. Quantum Electron. 2018, 25, 1-8. [CrossRef]

26. Guldager, B.; Brixen, K.T.; Jørgensen, S.J.; Nielsen, H.K.; Mosekilde, L.; Jelnes, R. Effects of Intravenous Edta Treatment on Serum Parathyroid Hormone (1-84) and Biochemical Markers of Bone Turnover. Dan. Med Bull. 1993, 40, 627-630. [PubMed]

27. Callis, G.; Sterchi, D. Decalcification of bone: Literature review and practical study of various decalcifying agents. Methods, and their effects on bone histology. J. Histotechnol. 1998, 21, 49-58. [CrossRef]

28. Madden, V.J.; Henson, M.M. Rapid decalcification of temporal bones with preservation of ultrastructure. Hear. Res. 1997, 111, 76-84. [CrossRef]

29. Feng, T.; Zhu, Y.; Wang, X. Functional Photoacoustic and Ultrasonic Assessment of Osteoporosis-A Clinical Feasibility Study. BME Front. 2020, 2020, 1081540. [CrossRef]

30. Cox, B.T.; Laufer, J.G.; Beard, P.C.; Arridge, S.R. Quantitative spectroscopic photoacoustic imaging: A review. J. Biomed. Opt. 2012, 17, 061202. [CrossRef]

31. Jacques, S.L. Optical properties of biological tissues: A review. Phys. Med. Biol. 2013, 58, R37. [CrossRef]

32. Wear, K.A. Mechanisms of Interaction of Ultrasound with Cancellous Bone: A Review. IEEE Trans. Ultrason. Ferroelectr. Freq. Control. 2020, 67, 454-482. [CrossRef]

33. Udelhoven, T.; Emmerling, C.; Jarmer, T. Quantitative analysis of soil chemical properties with diffuse reflectance spectrometry and partial least-square regression: A feasibility study. Plant Soil 2003, 251, 319-329. [CrossRef]

34. Taicher, G.Z.; Tinsley, F.C.; Reiderman, A.; Heiman, M.L. Quantitative magnetic resonance (QMR) method for bone and whole-body-composition analysis. Anal. Bioanal. Chem. 2003, 377, 990-1002. [CrossRef] [PubMed]

35. El Maghraoui, A.; Borderie, D.; Cherruau, B.; Edouard, R.; Dougados, M.; Roux, C. Osteoporosis, body composition, and bone turnover in ankylosing spondylitis. J. Rheumatol. 1999, 26, 2205-2209. [PubMed] 
36. Boskey, A.L.; Robey, P.G. The Composition of Bone. In Primer on the Metabolic Bone Diseases and Disorders of Mineral Metabolism; Wiley: Hoboken, NJ, USA, 2013.

37. Abdulsamee, N. Erbium Family Laser: Silent Revolution in Dentistry. Review. EC Dent. Sci. 2017, 13, 168-190.

38. Deán-Ben, X.; Gottschalk, S.; Mc Larney, B.; Shoham, S.; Razansky, D. Advanced optoacoustic methods for multiscale imaging of in vivo dynamics. Chem. Soc. Rev. 2017, 46, 2158-2198. [CrossRef] [PubMed]

39. Fantarella, D.; Kotlow, L. The 9.3- $\mu \mathrm{m} \mathrm{CO}_{2}$ Dental Laser: Technical Development and Early Clinical Experiences. J. Laser Dent. 2014, 22, 1.

40. Featherstone, J.; Nelson, D. Laser effects on dental hard tissues. Adv. Dent. Res. 1987, 1, 21-26. [CrossRef]

41. Bashkatov, A.N.; Genina, E.A.; Kochubey, V.I.; Tuchin, V.V. Saratov Fall Meeting 2005: Optical Technologies in Biophysics and Medicine VII; International Society for Optics and Photonics: Bellingham, WA, USA, 2006; Volume 6163.

42. Mejia, P.A.L.; Manuel, U.C.G.; Pérez, J.L.J.; Gomez, L.H.H.; Butron, H.L.; Cruz-Orea, A. Photoacoustic Spectroscopy Applied to the Study of Bone Consolidation in Fractures. In Materials Science Forum; Trans Tech Publications: Bäch, Switzerland, 2005; Volume 480, pp. 339-344.

43. Firbank, M.; Hiraoka, M.; Essenpreis, M.; Delpy, D. Measurement of the optical properties of the skull in the wavelength range 650-950 nm. Phys. Med. Biol. 1993, 38, 503. [CrossRef]

44. Van Veen, R.L.P.; Sterenborg, H.J.; Pifferi, A.; Torricelli, A.; Chikoidze, E.; Cubeddu, R. Determination of visible near-IR absorption coefficients of mammalian fat using time-and spatially resolved diffuse reflectance and transmission spectroscopy. J. Biomed. Opt. 2005, 10, 054004. [CrossRef]

45. Taroni, P.; Comelli, D.; Pifferi, A.; Torricelli, A.; Cubeddu, R. Absorption of collagen: Effects on the estimate of breast composition and related diagnostic implications. J. Biomed. Opt. 2007, 12, 014021. [CrossRef]

46. Ehrlich, H.; Koutsoukos, P.G.; Demadis, K.D.; Pokrovsky, O.S. Principles of demineralization: Modern strategies for the isolation of organic frameworks: Part II. Decalcification. Micron 2009, 40, 169-193. [CrossRef]

Publisher's Note: MDPI stays neutral with regard to jurisdictional claims in published maps and institutional affiliations.

(C) 2020 by the authors. Licensee MDPI, Basel, Switzerland. This article is an open access article distributed under the terms and conditions of the Creative Commons Attribution (CC BY) license (http://creativecommons.org/licenses/by/4.0/). 